\title{
Application research of physical ID in the whole process of disposal of waste materials in the State Grid
}

\author{
Yonghuan $\mathrm{Hu}^{1}$, Jian Wang ${ }^{2}$, Feng Xiao ${ }^{3}$, Fengna Dong ${ }^{3}$, Yongxu Zhang ${ }^{3, *}$ \\ ${ }^{1}$ State Grid Shanghai Company \\ ${ }^{2}$ State Grid Shanghai Jiading Power Supply Company \\ ${ }^{3}$ Shanghai Jiulong Enterprise Management Consulting Co. Ltd
}

Keywords: The physical ID, Waste materials management, Product life cycle management.

\begin{abstract}
In order to deepen the application of product life cycle management (PLM) in enterprise and realize the full-stage closed-loop standard of asset life cycle, to focus on the analysis of the key and difficult problems in the traditional waste material management process, to find out the application method of asset uniform identity code in the management of waste materials, to analyze its positive impact on the management of waste materials and overall asset management and information construction at the current stage. According to the application of physical ID at the current stage, the deep application of product life cycle management (PLM) in enterprise information construction is expected.
\end{abstract}

\section{Introduction}

Information technology to stimulate industrialization development strategy is a long-term 50year China enacted.At the same time, under the pressure of economic globalization, enterprise information has also become one of the main effective ways to enhance the core competitiveness of enterprises. In the application of enterprise information, Product Life Management (PLM) is the world's leading enterprise information thinking, is an open and interoperable solution that covers the entire life cycle of a product from birth to death. The key to building such an enterprise information environment is a systematic central product data repository that records all product information(Jiangnan, Qian,2007).Grid Asset unified identification code (Referred to as "physical ID") is refers to for the realization of the various stages of coding asset life management process, project code, project WBS coding, coding of materials, equipment and assets coding and code linkage information, is physical coding equipment, is the only and lifetime identity number of the object, is also the core of building a product data repository. So the further promoting the application of physical ID in the whole process of waste material management is particularly important for the improvement of the closed-loop management standard of asset life cycle management.

\section{Traditional way of disposal of waste materials}

As a typical asset-intensive company, State Grid enterprises with highly modern equipment and huge assets need to use asset life cycle management method for a large number of equipment and 
assets. It is of great significance to reduce costs and ensure efficient and safe operation of the power system (Jingjing, Zhou, 2013).Disposal of waste materials is an important part of product life cycle management. According to the latest State Grid Corporation waste materials management methods and work processes, the standardization process for waste disposal of power materials is broadly divided into five key links: decommissioning and exiting plans, decommissioning and exiting physical retirement approval, physical demolition and handover, exiting materials recycling, and reporting waste disposal.

Specific waste materials recycling and disposal process begins with the demolition principles and estimates determined by the asset unit according to the feasibility study stage, and prepares the project demolition plan, and reviews the scope, and content of the demolition plan, and clarifies the disposal method of the dismantled assets. For the waste materials, physical assets identification of storage units made an application to the physical asset management, and with the physical assets management, finance and other departments to handle the formalities of retirement. The waste was reported to be demolished under the supervision of the project manager, and was transported to the warehouse by the asset storage unit, and with the fixed assets technical appraisal and warehousing transfer order for storage with the Simultaneous approval process for fixed assets. After the approval is completed, the waste materials in the warehouse can be reported to the waste disposal. After the bidding transaction notice is issued, the storage unit of the waste material physical assets shall sign a contract for the sale of waste materials with the dealer of the contract according to the provisions of the company contract management. After receiving the full payment for the contract for the sale of used materials, the material company will organize the waste buyer to hand over the physical materials of the waste materials and complete the physical handover of the waste materials. At this point, the recycling and disposal of waste materials has basically ended. The materials have experienced a full life cycle consisting of external procurement, engineering construction, operation and maintenance, and decommissioning.

At present, there are some key and difficult problems in the processing of waste materials.

(1) Fragmented record of waste material recycling. It is very difficult to track the recycling process according to the single assets and engineering projects, which makes it impossible to realize the visual management of waste materials.

(2) There is a lack of effective control measures for the full recovery of waste materials, and there is a certain risk of asset loss.

(3) The material department's custody and disposal process of materials and the asset management department and project management department are not directly coordinated internally.

\section{The physical ID application is optimized in the whole process of waste materials}

In order to solve the problems existing in the traditional waste material management process and further reduce the cost of enterprise asset management and improve the efficiency of material circulation, this paper proposes the optimization scheme of physical ID to the whole process of waste management.

\subsection{The State Grid material physical ID}

The physical ID is the unified identity code of the grid asset, which follows the unified coding rule and consists of "company code segment + system generation identification code + serial number + check code”, which is the key part of promoting the whole life cycle of the asset.

In the actual application stage of the physical ID, the physical ID may use a two-dimensional code, an RFID tag or the like as a carrier according to different physical characteristics of the asset, installation environment and the like. The incremental asset physical ID tag is installed during the 
material procurement phase. The inventory asset physical ID tag is installed during the operation and maintenance phase. Through the construction of physical ID of grid assets, the unite of asset life-time identity coding is promoted, and the information assets are efficiently shared and shared during the whole life cycle of planning, procurement, operation and maintenance, and decommissioning, and the asset management level is improved.

\subsection{The optimization application of physical ID in the whole process of disposal of waste materials}

\subsubsection{The decommissioning of waste materials}

The physical use and storage department applies for the decommissioning of the equipment by scanning the physical ID. Automatically generate a decommissioned asset demolition list based on the obtained waste materials information and application information and push it to the ERP system. This optimization process has greatly reduced the time for the application process for the retirement of waste materials, and greatly reduced the communication costs within the enterprise.

\subsubsection{The process of dismantling waste materials}

The project management department and the physical use and storage unit will check the physical assets of the decommissioned power grid by scanning physical IDs, check the accuracy of the demolition equipment, and establish the image and image data and actual recovery schedule of the on-site waste materials removal process. Database, so that the entire process of visual management of the dismantling process can be realized

\subsubsection{The process of warehousing of waste materials}

The physical storage unit and the material department handle the transfer and warehousing procedures of waste materials. They scan the physical ID label, verify the basic information of waste materials, obtain the code of waste materials, and complete the storage of waste materials. Simultaneous handover of waste materials and physical ID. While greatly reducing the verification of material information, it also improves the accuracy of information matching, and reduces the cost of waste material management of enterprises to a certain extent.

\subsubsection{The sales process of waste materials}

All levels of material companies (supply centers) create sales orders for waste materials in the system, and record physical ID information in sales orders.

\subsubsection{The waste materials from the session}

According to company materials waste materials disposal transaction notice, organize buyer, raw materials owned unit signed a contract of sale of waste materials, waste materials and the final completion of the physical transfer of a single handle. Under the witness of the inspectors, the material company, the original asset management unit, and the buyer jointly counted and weighed, and all the people handed over the data. Finally, the material company scanned the physical ID tag of the equipment to complete the ERP system out of the warehouse. The relevant personnel of the material company are responsible for maintaining the state of payment of the contract for the sale of waste materials in the ERP system. The physical ID makes the verification information time in the handover process greatly reduced, improves the circulation efficiency of waste materials, and reduces the asset management cost of the enterprise. 


\subsubsection{The monitoring process of the whole process of waste materials disposal}

The system automatically collects the information of each process node of waste materials disposal, and tracks the disposal status of the decommissioned assets with the physical ID as the main line, and displays the physical ID as the unique identity information in a unified visual screen. The entire process of waste material management is visualized.

\subsubsection{The recycling of waste materials}

The system automatically calculates the physical ID information of waste materials, matches all asset management items in the system, and automatically generates a list of equipment that can be reused for used materials. The system performs a second depth calculation match on the physical ID information in the equipment list and the corresponding asset item to make a final confirmation. Optimizing the recycling of waste materials has greatly improved the re-use rate of materials and the correct rate of material matching, reduced the time for review and matching, and greatly improved the efficiency of asset management (Wanrong, Xu, 2009).

The application of physical ID technology in the four main links of "recycling, warehousing, auction, and delivery" of waste materials and the application of physical ID in the external collaboration between various departments of the enterprise and external supplier buyer. It has realized the full-process closed-loop online visual control in the whole process of power grid waste disposal management, improved the disposal efficiency of waste materials, reduced the cost of material management, and established a data knowledge base based on physical ID as the basis of asset life cycle. An important part of the process completes closed-loop intelligent management and control, and also reflects the practical significance of asset life cycle management.

\section{Summary and outlook}

The construction of physical ID in the whole process of waste material management solves the key and difficult problems existing in the traditional model, and further improves the closed-loop standard of product life cycle management in enterprise asset management, and produces corresponding positive impact.

At the same time, in order to realize the all-round information management of enterprise asset management, the application of physical ID in the whole process of waste material management is further prospected here.

\subsection{New technology application}

Realize the use of RFID or tag information to automatically enter waste materials into the system: Write important information such as asset number, equipment number, and warehousing date of scrapped fixed assets into RFID, or record them into ERP system through labels. At the same time, the inventory of waste materials is counted by means of RFID or label scanning.

\subsection{Establish an economic analysis model for decommissioning equipment}

The physical asset value management department conducts economic analysis on the planned decommissioning and dismantling equipment involved in the power grid reconstruction project.

Retirement asset income value $=$ depreciation of decommissioned assets + operation and maintenance fee - storage logistics cost.

Among them, depreciation of decommissioned assets $=$ original value of assets $*$ pricing depreciation rate. 
Operation and maintenance fee $=$ net asset value $*$ operation and maintenance rate $=$ original value of assets * (1-n years * pricing depreciation rate) $*$ operation and maintenance rate.

When the income of the decommissioned assets in the economic analysis model is negative, that is, negative income, the equipment should be scrapped; when the return value of the decommissioned assets is positive, that is, positive income, the equipment proposal may not be disposed of.

\subsection{Forecast of waste storage capacity demanded by physical ID assets}

Strengthen the monitoring of the new rate of demolition equipment and the forecast of the amount of old equipment in the inspection and inspection. Based on the common storage standards for decommissioning equipment, improve the forecasting ability of future decommissioning assets (new rate, quantity and required storage capacity) and create an existing warehouse storage monitoring and early warning system, and finally realize the optimization of decommissioning materials disposal strategy.

\section{References}

[1] Jiangnan, Qian, 2007, A Summary of Research on Asset Life Cycle Management, China Electric Power Education. 10, pp. 111-112

[2] Jingjing, Zhou, 2013, Research on Life Cycle Management of State Grid Assets, Times economy. 19, pp.48-52

[3] Wanrong, $X u$, 2009, LCM events for asset retirement and decommissioning equipment reuse, Shanghai Electric Power. 6, pp.503-507 